\title{
Displacement damage effects in silicon MEMS at high proton doses
}

\author{
João Gomes and Herbert R. Shea \\ Ecole Polytechnique Fédérale de Lausanne, Microsystems for Space Technologies Laboratory, CH- \\ 2002 Neuchâtel, Switzerland
}

\begin{abstract}
We report on a study of the sensitivity of silicon MEMS to proton radiation and mitigation strategies. MEMS can degrade due to ionizing radiation (electron-hole pair creation) and non-ionizing radiation (displacement damage), such as electrons, trapped and solar protons, or cosmic rays, typically found in a space environment. Over the past few years there has been several reports on the effects of ionizing radiation in silicon MEMS, with failure generally linked to trapped charge in dielectrics. However there is near complete lack of studies on displacement damage effects in siliconMEMS: how does silicon change mechanically due to proton irradiation? We report on an investigation on the susceptibility of $50 \mu \mathrm{m}$ thick SOI-based MEMS resonators to displacement damages due to proton beams, with energies from 1 to $60 \mathrm{MeV}$, and annealing of this damage. We measure ppm changes on the Young's modulus and Poisson ratio by means of accurately monitoring the resonant frequency of devices in vacuum using a Laser Doppler Vibrometer. We observed for the first time an increase (up to 0.05\%) of the Young's modulus of single-crystal silicon electromagnetically-actuated micromirrors after exposure to low energy protons (1-4 MeV) at high absorbed doses $\sim 100$ Mrad (Si). This investigation will contribute to a better understanding of the susceptibility of silicon-based MEMS to displacement damages frequently encountered in a space radiation environment, and allow appropriated design margin and shielding to be implemented.
\end{abstract}

Keyword: MEMS reliability, radiation effects, displacement damage, NIEL, mechanical effects

\section{Introduction}

The reliability of Micro-Electro-Mechanical System (MEMS) devices for many applications on Earth is well established. However the effects of space radiation on MEMS is a field where to date there has been limited research. In part, the lack of studies on both mechanical reliability and radiation sensitivity of MEMS has contributed to limit their applicability as systems to be flown in space craft systems. In a space environment, MEMS like their electronic counterparts can degrade due to ionizing radiation (total ionizing dose) and non-ionizing radiation (displacement damage dose), due to the influence of particles such as electrons, protons, or cosmic rays, typically found in a space radiation environment. Recent investigations [1-4] performed on accelerometers and RF switches have showed that these devices exhibit dramatic changes on their operation when exposed to ionizing doses as low as $30 \mathrm{krad}$. On the other end, microengines tested at Sandia National laboratories, in Albuquerque, New Mexico, showed evidence of device survivability up to $10 \mathrm{Mrad}$, and for some devices even up to $1 \mathrm{Grad}$ [3]. For devices that operate on the electrostatic principle and have dielectric layers directly exposed to the radiation beam failures are generally attributed to ionizing radiation effects due to radiation induced-trapped charge that can lead to offsets on the electrical fields that govern the motion of the devices or even total failure of the component due to stiction.

An important goal of radiation studies is to understand and correlate the effects of different types of radiation with the properties of materials and devices. MEMS components designed for Low-earth-orbit (LEO) applications, as components for satellites for example are routinely exposed to high proton fluences that can be detrimental to the device due to their ability to produce both ionizing and non-ionizing effects as they penetrate the material. At present the

Reliability, Packaging, Testing, and Characterization of MEMS/MOEMS and Nanodevices X,

edited by Sonia Garcia-Blanco, Rajeshuni Ramesham, Proc. of SPIE Vol. 7928, 79280G

(C) 2011 SPIE · CCC code: 0277-786X/11/\$18 $\cdot$ doi: 10.1117/12.873546

Proc. of SPIE Vol. 7928 79280G-1 
mechanisms of damage due to ionizations are reasonably well understood [5]. However, there is near complete lack of studies on displacement damage effects in silicon-MEMS due to the effects of particles such as protons.

In order to evaluate the susceptibility of silicon based-MEMS devices to displacement damages, and which parameters influence it, we have exposed commercial single-crystal silicon based micromirrors to protons (at different energies) and considerable high total ionizing dose $\sim 100 \mathrm{Mrad}(\mathrm{Si})$. The main objective of this work was to determine a dose where displacement damage effects could be observed on the crystal lattice of the silicon material. As described in section 2.2 and 2.3 changes on the material properties of the silicon (e.g. Young's modulus) were monitored by measuring the resonant frequency of devices before and after exposure. In section 2.4 we describe a methodology that allows calculating the ionizing and non-ionizing doses absorbed by the devices using a numerical code SRIM. In section 3 , we describe briefly an on-going investigation that aims to determine on the susceptibility of silicon to displacement damages due to protons $(10-60 \mathrm{MeV})$. For this purpose we present single-crystal based cantilever test structures that were fabricated using a silicon-on-insulator process for investigation of displacement damage in silicon at energies relevant to the space environment.

\section{Experimental procedure}

\subsection{Test Devices}

Single-crystal silicon micromirrors (manufactured by Lemoptix [6]) were employed in this work to investigate the susceptibility of silicon to displacement damages due to protons in the energy range 1-5 MeV. The micromirrors are formed by a suspended square mass plate attached to two torsional beams at the chip far ends (see Fig 1). On the top of the mirror-plate a metal track is deposited for excitation purposes. Electromagnetic actuation of the device is performed by sitting the entire chip $(3.5 \times 2.55 \times 0.4 \mathrm{~mm})$ on top of an arrangement of permanent magnets and exciting the device using an appropriate AC signal using the appropriate bond pads. In this work we use micromirror with two different operational areas: $0.43 \times 0.43 \mathrm{~mm}^{2}$ (type I) $0.9 \times 0.9 \mathrm{~mm}^{2}$ and (type II), respectively. Following fabrication, each micromirror is glued to a PCB and wire bonded for testing. The structural silicon layer of the device is $30 \mu \mathrm{m}$ thick.

Figure 1 shows an optical micrograph of the top view of one type I device. The micromirror formed by a square plate including its metal tracks and torsional spring beams are clearly seen on the image. For the device shown in Figure 1 the resonant frequency is near $17 \mathrm{kHz}$. The optical scan angle at resonance is found to be $+/-15^{\circ}$ for an excitation voltage of $8.3 \mathrm{Vp}-\mathrm{p}$ (53 mA rms current consumption). For this work one micromirror type I and one type II were utilized.

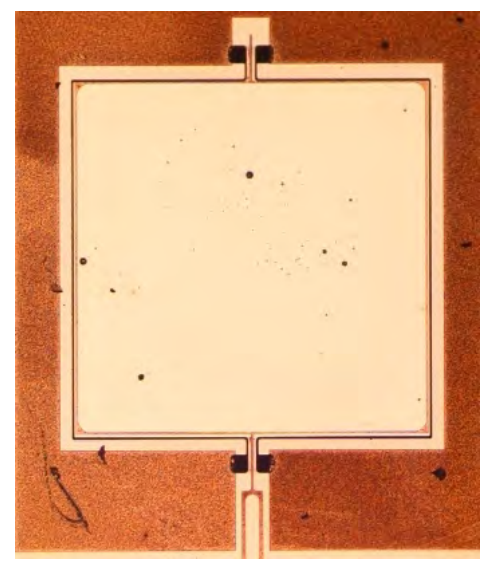

Figure 1: Optical image of top view of one of the electromagnetic actuated MEMS mirrors used for testing on this work (Lemoptix micromirror, [6]). 


\subsection{Measurement Technique}

Resonant frequency measurements focused on measuring the frequency of vibration of the tilting mode of the micromirrors (see Fig 1). As shown in Figure 2, the test technique comprised a Laser Doppler Vibrometer (MSV-400 from Polytec), a Keithley multimeter (2100 digital multimeter), an external function generator (Agilent 33220A), a HV amplifier and a pc running LabView 2008. A typical measurement would involve placing the device under the optics of the vibrometer and excite its resonance tilting mode using the external function generator (sine wave signal, $\mathrm{V}_{\mathrm{in}}=1 \mathrm{~V}_{\mathrm{p}-\mathrm{p}}$ ). The LDV measures the velocity of a moving object in the time domain. If, as is possible with the MSV-400, the time domain data is converted to the frequency domain, the resolution of the measurement is ultimately limited by the number of FFT lines available for the measurement. For a frequency bandwidth of $20 \mathrm{kHz}$ and using maximum number of lines available (6400 FFT lines) the resolution on the frequency measurement can calculated to be $3.1 \mathrm{~Hz}$. Since we needed better accuracy, we read the output signal (voltage proportional to velocity) of the vibrometer with a Keithley multimeter and sweep the frequency using a custom-implemented LabView code to get a velocity vs. frequency plot. This way we could achieve a frequency resolution better than $0.5 \mathrm{~Hz}$, limited principally by the Q factor of the device. A schematic representation of the technique is shown in Figure 2 (a) while in (b) we show the result from frequency measurement performed on a device type I. Using this technique, the resonant frequency of each specimen was determined before and after irradiation. Frequency shifts could then be related to changes on the material properties of the silicon material. Frequency measurements were performed in air and in vacuum ( $\mathrm{P}=0.1 \mathrm{mbar}$ ) using a test chamber (an image of the test chamber can be seen in Figure 6).

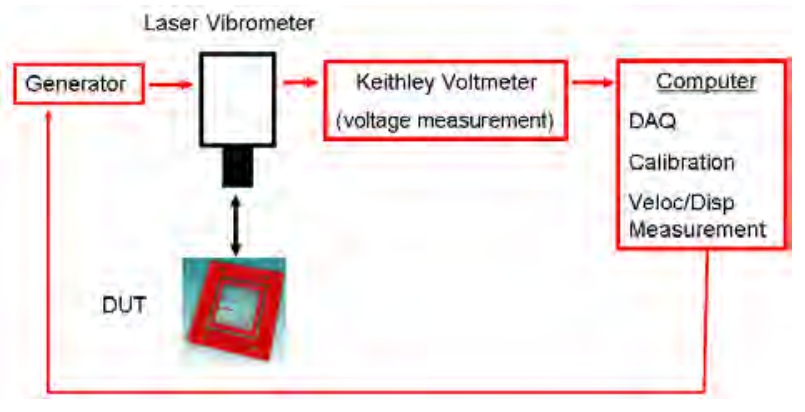

(a)

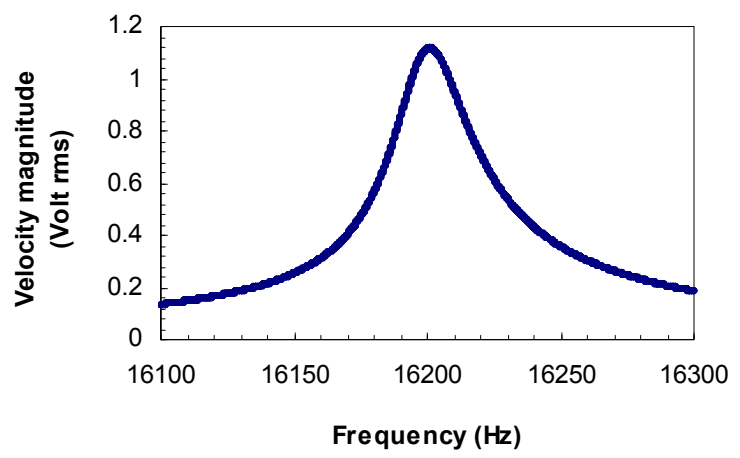

(b)

Figure 2: (a) Schematic representation of the setup used for the frequency measurements (b) Typical resonance response curve obtained for Lemoptix micromirror type I.

\subsection{Dose calculation using SRIM}

The Stopping and Range of Ions in Matter (SRIM code developed by James F. Ziegler, [7]) is a Monte Carlo code that simulates the transport of energetic ions in materials thus allowing for the calculation of parameters such as vacancy production rates or project ranges in a variety of substrate materials. In this work we have used SRIM to calculate the total ionizing (Dose ionize $(\mathrm{t})$ ), non-ionizing absorbed doses $\left(\right.$ Dose $\left._{\text {displ }}(\mathrm{t})\right)$ and projected ranges for protons in silicon. The calculation of total ionizing absorbed doses for protons in silicon is dependent on the determination of a parameter called linear energy transfer function (LET) which gives us a measure of the ionization energy loss rates as the particle penetrates in the material and is readily available from the SRIM output file during ranges/table distributions. The product of this parameter and the particle fluence yields the total ionizing dose absorbed by the device. A more detailed 
procedure is necessary for calculation of the absorbed dose due to non-ionizing effects. The procedure employed was similar to the one described in [8] and relied on obtaining with SRIM the "vacancy.txt" output files detailing the number of vacancies produced for both the incident particle and resultant recoils, which were processed and allowed conversation of the vacancy formation rates to NIEL using the Kinchin-Pease relationship. Figure 3 below shows the LET and NIEL results obtained from several SRIM simulations for proton energies of $1 \mathrm{MeV}, 4.3 \mathrm{MeV}, 10 \mathrm{MeV}$ and 60 $\mathrm{MeV}$ as a function of the penetration depth in silicon. Using the calculated values and multiplying them by the particle fluence we can calculate the ionizing and non-ionizing absorbed doses following the expressions,

$$
\begin{aligned}
& \text { Dose }_{\text {ionize }}(t)=1.6 \times 10^{-8} \times L E T(t) \times F \\
& \text { Dose }_{\text {displ }}(t)=1.6 \times 10^{-8} \times \operatorname{NIEL}(t) \times F
\end{aligned}
$$

where, Dose ionize $(t)$ and Dose displ $(t)$ are the ionizing and non-ionizing doses as a function of the depth of the material, in the unit of rad; $t$ is the depth of the material, in micron; $1.6 \times 10^{-8}$ is a unit conversion parameter, in the unit of rad $\mathrm{g} / \mathrm{MeV}$; LET(t) and NIEL(t) are the ionizing and non-ionizing energy loss as a function of the depth of the material, in the unit $\mathrm{MeV} \mathrm{cm} 2 / \mathrm{g} ; \mathrm{F}$ is the fluence of the incident protons, in proton $/ \mathrm{cm}^{2}$. In this work, we used Eqs. 1 and 2 above and results obtained in Figure 3 (a) and (b), we have calculated the ionizing and non-ionizing doses for the exposures using the Lemoptix devices (structural silicon layer $\sim 30 \mu \mathrm{m}$ thick). For exposures with $4.3 \mathrm{MeV}$ protons, for example, the ionizing and non-ionizing doses at $\mathrm{t}=30 \mu \mathrm{m}$ were calculated to be $100 \mathrm{Mrad}(\mathrm{Si})$ and $30 \mathrm{krad}$ ( $\mathrm{Si}$ ), respectively. Dose calculation for exposures at 10 and $60 \mathrm{MeV}$ are reported in section 3.3.

Further, the projected ranges of protons in silicon could be obtained using the stopping/range tables sub-routine with SRIM.

For proton energies of $1 \mathrm{MeV}, 4.3 \mathrm{MeV}, 10 \mathrm{MeV}$ and $60 \mathrm{MeV}$ the projected ranges were calculated as $16.3 \mu \mathrm{m}, 167.4$ $\mu \mathrm{m}, 709.2 \mu \mathrm{m}$ and $16.8 \mathrm{~mm}$, respectively. Since the devices are directly exposed to the proton beams during testing, i.e. no shieldings are involved, we expect that for $1 \mathrm{MeV}$ protons the particles will be stopped inside the active region of silicon while for $4.3 \mathrm{MeV}$ the protons would flow through the devices (structural layer of the Lemoptix micromirror 30 $\mu \mathrm{m}$ thick). For proton beams with kinetic energies of 10 and $60 \mathrm{MeV}$ (relevant for the investigation in section 3.3) we can safely assume that protons will flow though the thickness of the devices as the structural layer of the fabricated cantilevers is $50 \mu \mathrm{m}$ thick.
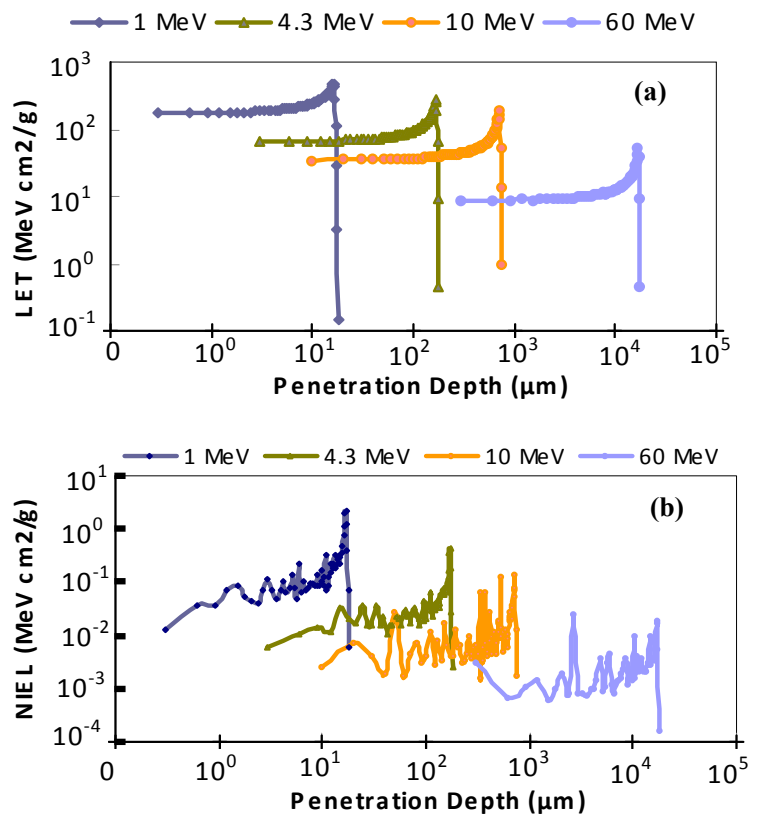

Figure 3: (a) LET and (b) NIEL as a function of penetration depth for protons in silicon computed using SRIM. 


\section{Results and Discussion}

\subsection{Proton irradiation at 1 and $4.3 \mathrm{MeV}$}

In this section we present the results from the irradiation ( 2 devices were irradiated) of Lemoptix micromirrors exposed to protons $(1 \mathrm{MeV}$ and $4.3 \mathrm{MeV})$. The devices were irradiated at the $\mathrm{CN}$ Van de Graff accelerator of the Laboratori Nazionali di Legnaro of the INFN, Italy. The main motivation for this work dealt with finding a suitable absorbed dose at which mechanical changes due to proton beam exposures could be observed in single-crystal silicon. To the knowledge of the authors there is limited data on the mechanical damage or displacement damages in silicon due to protons at scales relevant to MEMS. This study also motivated the more complete study with cantilevers described in section 3.3.

For irradiation one device of type I and one type II (see Figure 1) were subjected to proton beams with kinetic energies of $1 \mathrm{MeV}$ and $4.3 \mathrm{MeV}$, respectively. The devices were exposed to a total ionizing absorbed dose of $100 \mathrm{Mrad}(\mathrm{Si})$ and during irradiation the device was always normal to the beam. The dose rates for the testing were $22 \mathrm{krad} / \mathrm{s}$ and $43 \mathrm{krad} / \mathrm{s}$, for device type I and II, respectively. For measurements, the resonant frequency was calculated from the lorentzian curves (Figure 2b) by using a fitting algorithm. As shown in Figure $2 \mathrm{~b}$ for a device tape $\mathrm{I}$, before irradiation, the resonant frequency was found to be $16197.94 \mathrm{~Hz}$. The quality factor of the micromirrors is also readily available from measurements performed at the $3 \mathrm{~dB}$ points of the curve and, for this particular device, was found to be $\mathrm{Q} \sim 463$ (in air) and $\mathrm{Q}=1145(\mathrm{P} \sim 0.1 \mathrm{mbar})$.

In Figure 4 (a) and (b) we show the typical behavior of the resonant frequency (for measurements performed in air) after irradiation over a period of 23 days. Observation of the curves show evidence of shifts on the frequency of the devices: in the period immediately after irradiation the resonant frequency of the devices was found to increase followed by a decrease possibly due to annealing of the damage on the period thereafter. In particular, for the specimen type I (Figure 4a), we observed an increase on the resonance frequency in the period immediately after the irradiation (frequency shift $7.8 \mathrm{~Hz}$ ). Continued measurements showed that the resonant frequency of the device decreased (from day 3 to day 9) to a value closer to the frequency before irradiation. Measurements performed on the specimen 23 days after irradiation showed no difference in frequency measurements compared to previous measurement (difference in frequency within the resolution of the measurement $0.5 \mathrm{~Hz}$ ). This result suggests that due to exposures bulk defects were introduced in the material, presumably changing the material properties of the crystal lattice. It is interesting to note that for the specimen type II (Figure 4b) we observed the same behavior although to a lesser extent (frequency shift of $1.84 \mathrm{~Hz}$ ). The difference in frequency shifts is still unclear however for both devices we measured a total recovery in frequency after a total of 23 days. This result can be explained if we consider that proton exposures induce defects in the material (rather than permanent damage). Since these defects can be mobile even at room temperature, during the period after irradiation, they can re-organize themselves in the crystal lattice so that the systems reach equilibrium thus leading to the total recovery on the frequency observed. This annealing behavior is commonly seen in radiation damage experiments, with time constant from seconds to months.

Lastly, we have measured the resistance of the devices, 2 days, 9 days and 20 days after irradiation. Comparison of those values with the measurements before irradiation showed that this parameter did not change within the experimental error for the measurement.

Based on the measured shifts in resonant frequency and ignoring any changes on temperature and pressure during measurements, we report on this work induced changes on the Young's modulus of the single-crystal silicon material due 
to exposure to protons (1-4.3 MeV energy range) of the order of $0.05 \%$. To the knowledge of the authors this is the first time that such changes are reported for MEMS due to exposures to proton beams.

In the next section (section 3.2) we present results from an investigation of thermal effects in silicon where we concluded on the dependence of the resonant frequency of single-crystal silicon MEMS on the measurement temperature. The dependency of the Q-factor of the devices on the environment pressure is also presented.

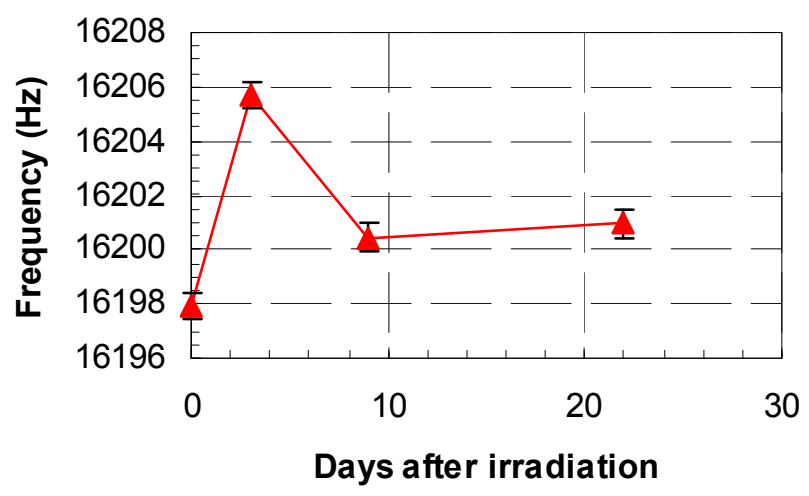

(a)

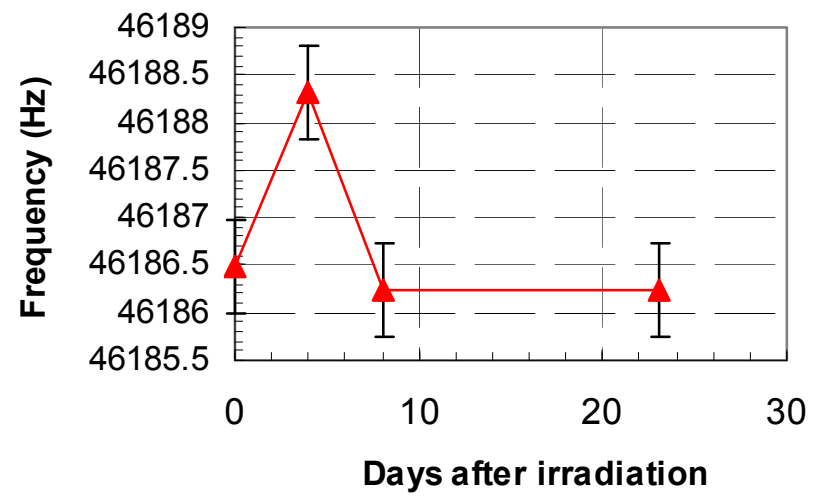

(b)

Figure 4: (a) and (b) Resonant frequency shifts as a function of irradiation day for devices type I and II, respectively. The first data point (at irradiation day zero) corresponds to the value of the frequency of the device before irradiation. The change in resonance

frequency indicates an increase in Young's modulus that anneals out after a few days. All frequency measurements were performed in air.

\subsection{Effect of pressure and temperature}

In order to determine the dependence of resonant frequency on the temperature during measurements, silicon cantilevers were fabricated using a silicon-on-insulator process, with nominal dimensions: $2000 \mu \mathrm{m}$ long, $80 \mu \mathrm{m}$ wide, $50 \mu \mathrm{m}$ thick. An optical image of one of the fabricated chips can be seen in Figure 7. We excited the first out-plane mode of resonance of the cantilevers using electrostatic actuation and measured the resonant frequency using the setup described in section 2.3. For measurements a controlled environment was achieved by fixing the silicon chips to a custom-built heater and placing the whole component inside a test chamber. The pressure during measurement was kept constant at $\sim 0.1$ mbar. The temperature inside the chamber could be varied in the range 29 to $51{ }^{\circ} \mathrm{C}$ and was monitored using a temperature meter (Omega HH12A, resolution $0.1^{\circ}$ ). An image of the test chamber is shown in Figure 6.

Figure 5 shows the results from the measurement. It is clear that the resonance frequency of the specimen (normalized to the frequency at $\mathrm{T}=28.9{ }^{\circ} \mathrm{C}$ ) decreases monotonically with the increase of temperature. Using beam bending mechanical analysis the resonant frequency for the first out-plane of a straight beam rectangular cantilever can be calculated following Eq. 3 [9],

$f=\frac{t}{4 \pi}\left(\frac{1.8751}{L}\right)^{2} \sqrt{\frac{E}{3 \rho}} \quad($ Eq. 3$)$ 
Where $f$ is the resonant frequency of the 1st out-plane mode of the cantilever, $L$ is the length of the cantilever, $E$ the Young's modulus of the material, $\rho=2330 \mathrm{~kg} / \mathrm{m}^{3}$ the density of the silicon, and $t$ the thickness of the device layer. From this expression we conclude that there are mainly two temperature dependent parameters that can influence the observed changes on the frequency of the specimen: the Young's modulus of the material and the thermal expansion of the silicon. If we define the linear coefficient of thermal expansion of the silicon material as $\alpha_{\mathrm{Si}}=2.6 \times 10^{-6} /{ }^{\circ} \mathrm{C}[10]$, the resonant frequency temperature coefficient, $\alpha_{\text {fres }}$, and the Young's modulus temperature coefficient, $\alpha_{\mathrm{E}}$, then following Eq. 3 we can write the relation between the coefficients as $2 \alpha_{\mathrm{f}} \sim \alpha_{\mathrm{Si}}+\alpha_{\mathrm{E}}$. Substituting for the values shown in Figure 5a and considering a temperature change of about $22.1^{\circ} \mathrm{C}$ we could calculate the Young's modulus temperature coefficient to be $-2.2 \times 10^{-4} /{ }^{\circ} \mathrm{C}$. Comparison of the rate change of Young's modulus with temperature with values in the literature [10] shows that the value obtained on this work is about 1.7 times higher than the one presented by the authors above. However, this difference can be explained if we consider that the temperature measurement during the experiment was a little underestimated due to the fact that measurements were performed inside the chamber rather than at specimen location.

Moreover, it is interesting to note that due to the high sensitivity observed for frequency measurements on the temperature, the resonant frequency of a single crystal silicon device is able to vary by $\sim 8 \mathrm{~Hz}$ for a temperature shift of only $\sim 5^{\circ} \mathrm{C}$. However, as shown in Fig. 4 frequency measurements performed on the same day for two different specimens resulted in different frequency shifts measured using the same experimental setup and specimen mounting technique. Additionally, measurements performed within a 14 day gap on two different specimens resulted on the same behavior, i.e. a complete recovery of the resonant frequency of the specimen to the same value as before irradiation. Based on this results it is the unlikely that the observed frequency shifts can be explained solely by changes on temperature. However, the results presented on this section suggest the environment temperature should be controlled during measurement. These preliminary results motivated the ongoing work that we outline in section 3.3, where for testing the resonant frequency measurements are performed at a fixed temperature $\left(45^{\circ} \mathrm{C} \pm 0.1{ }^{\circ} \mathrm{C}\right)$.

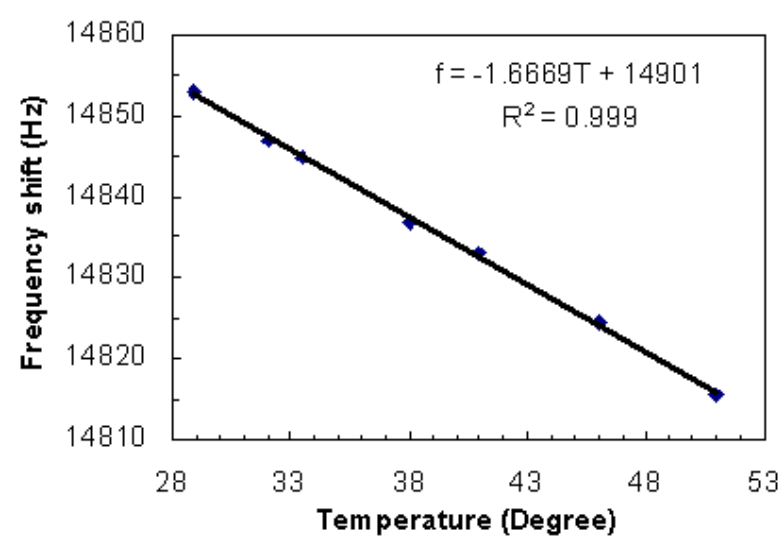

(a)

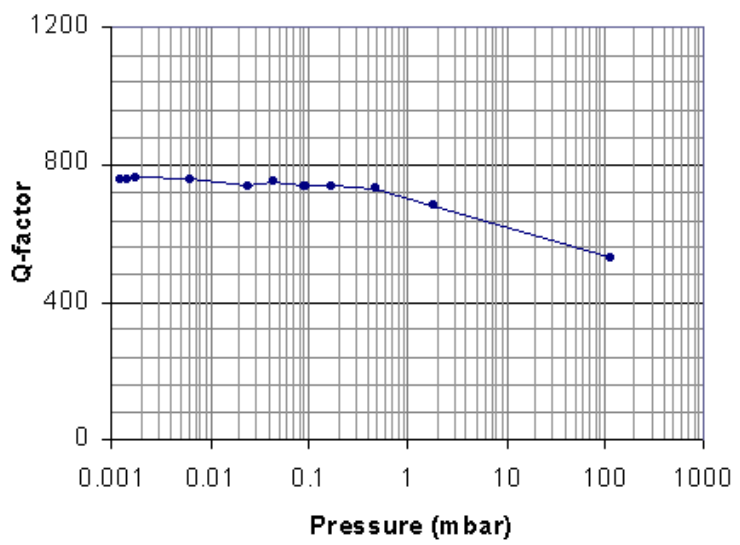

(b)

Figure 5: (a) The variation of the resonant frequency of the SOI cantilever as a function of temperature (in the range 28 $51{ }^{\circ} \mathrm{C}$ ); (b) Variation of the quality factor of the devices as a function of pressure in the test chamber.

Lastly, the quality factor (Q-factor) of silicon cantilever specimens was also determined at different pressures using the test chamber shown in Fig. 6. The overall aim of this work was to find a pressure range where air damping would be minimal and weakly pressure dependent so that we can determine a pressure region for reproducible measurements 
during testing. Cantilever test specimens were subjected to different pressures inside a vacuum chamber (pressure range $1.2 \times 10^{-3}-115 \mathrm{mbar}, 31.5 \pm 0.2^{\circ} \mathrm{C}$ ) and their Q-factor measured. Results from this investigation are shown in Figure 5 (b). As expected, the Q-factor of the specimens decreases as more air is added to the chamber, attaining of value of about 530 at nearly atmospheric pressure. However, we found that the Q-factor of the cantilevers remains constant for pressures in the range $1.2 \times 10^{-3}-6.2 \times 10^{-3}$ mbar. As shown in Figure $5 \mathrm{~b}$, within this pressure range the Q-factor of the cantilevers is constant $(\mathrm{Q} \sim 758)$ and we can therefore conclude that air damping does not play a significant role so that only material losses are possible in the system. Similarly to the temperature measurements, these results suggests that in order to increase the measurement accuracy for the investigation of radiation effects using the silicon MEMS fabricated for this work frequency measurements should be performed in the pressure range $1.2 \times 10^{-3}-6.2 \times 10^{-3}$ mbar.

Based on the results described on this section, we redesigned the test protocol so that we can filter out the effects of temperature and pressure from the frequency measurements. In this proton irradiation campaign we will follow the following protocol: 1) Place specimen inside chamber and pump the system to high vacuum $\left.\left(10^{-3} \mathrm{mbar}\right) ; 2\right)$ Pre-bake specimen at 110 degree for $25 \mathrm{~min} ; 3$ ) Decrease temperature to $45^{\circ} \mathrm{C}$ and perform measurements of resonant frequency for the different cantilevers on the chip with pressure in the range $1.2 \times 10^{-3}$ mbar to $6.2 \times 10^{-3}$ mbar; 3) Vent the chamber; 4) Place another specimen into the chamber and repeat sequence.

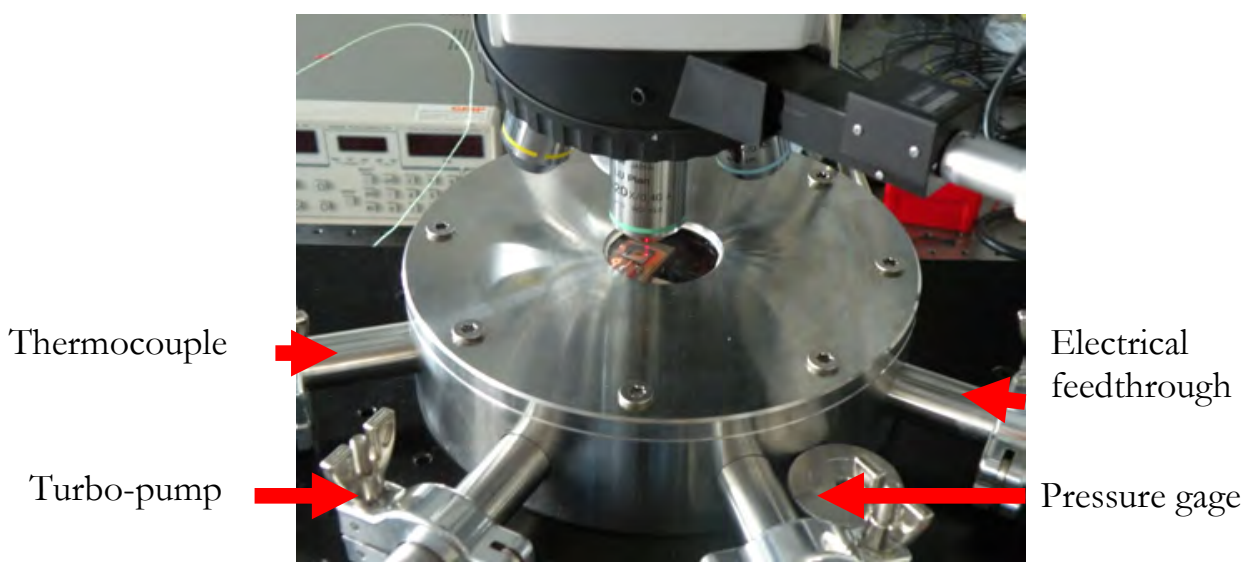

Figure 6: Close-up of the test chamber for specimen frequency measurements in a controlled environment

\subsection{Proton irradiation at 10 and $60 \mathrm{MeV}$}

We are currently investigating the susceptibility of single crystal silicon to proton radiation beams in the energy range $(10-60 \mathrm{MeV})$. For this purpose, single-crystal silicon cantilever specimens were fabricated using a silicon-on-insulator process (structural layer of the device $50 \mu \mathrm{m}$ thick) and irradiated at the OPTIS facility at Paul Scherrer Institute, Villigen, Switzerland. Figure 7 (a) and (b) shows two of the fabricated chips composed by cantilevers with lengths ranging from 300 to $2000 \mu \mathrm{m}$, and paddle resonators $\left(800 \mu \mathrm{m}\right.$ long, paddle area $\left.0.18 \mathrm{~mm}^{2}\right)$ for measuring changes on the material properties (Young's modulus and Poisson ratio) of the material due to the exposure to protons. 
For the irradiations, a number of fluence steps in the range $10^{11}-10^{15} \mathrm{p} / \mathrm{cm}^{2}$ were chosen. This work builds on the investigation described in section 3.2 that suggests threshold fluence for testing of the order $\sim 10^{13} \mathrm{p} / \mathrm{cm}^{2}$. Testing at high fluences means higher doses are expected, however the range of fluences proposed also covers the values typically found in a space environment. Following the work described in section 2.3, we calculated the total ionizing and non-ionizing doses, at the back end of the device $(t=50 \mu \mathrm{m})$ for proton energies of $60 \mathrm{MeV}$ to be $1.37 \mathrm{Mrad}(\mathrm{Si})$ and $563.9 \mathrm{rad}(\mathrm{Si})$ for a total fluence of $10^{13} \mathrm{p} / \mathrm{cm}^{2}$. This investigation is underway and testing will be performed following the test setup presented in section 2.2 using the protocol outlined on section 3.2.
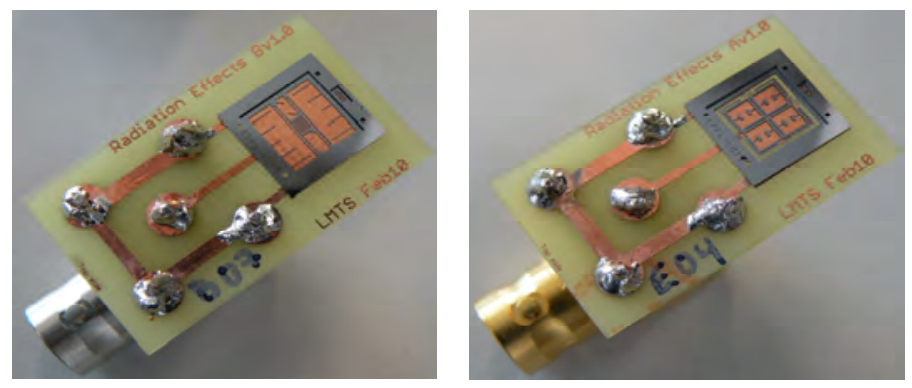

Figure 7: Optical imaging of the silicon chips composed by cantilever test specimens for radiation measurements fabricated using a silicon-on-insulator process. The specimens are mounted on a PCB for excitation and routing.

\section{Conclusion}

In this paper we reported preliminary results on bulk damage in silicon MEMS micromirrors due to exposure to proton beams with low kinetic energies $(1$ and $4.3 \mathrm{MeV})$. An experimental test setup was presented that allows for measuring shifts on the resonant frequency of the devices with a resolution better than $0.5 \mathrm{~Hz}$. Micromirrors tested at $1 \mathrm{MeV}$ protons exhibited an increase of frequency during the days immediately after the irradiation, where frequency shifts up to $8 \mathrm{~Hz}$ have been observed. The resonant frequency shifts are believed to be related to material changes in silicon due to exposure of the devices to proton radiation beams. Based on the data obtained and ignoring any changes on temperature and pressure during measurements, we report on this work induced changes on the Young's modulus of the single-crystal silicon material due to exposure to protons (1-4.3 MeV energy range) of the order of $0.05 \%$. To the knowledge of the authors this is the first time that such changes are reported for MEMS due to exposures to proton beams. However, in this paper we also investigated thermal and pressure effects on the resonant frequency and Q-factor of the devices using silicon-based cantilever structures fabricated using a silicon-on-insulator process. Results from measurements show that the frequency of the devices decreases monotonically with temperature thus inducing a decrease on the Young's modulus of the material $\left((\Delta \mathrm{E} / \mathrm{E}) / \Delta \mathrm{T} \sim-2.2 \times 10^{-4} /{ }^{\circ} \mathrm{C}\right)$. A suitable region for measurements, where the Q-factor of the devices presents a weak dependence of the environment pressure was determined: $1.2 \times 10^{-3} \mathrm{mbar}$ to $6.2 \times 10^{-3} \mathrm{mbar}$. A test protocol is proposed for continuing the investigation using single-crystal silicon cantilevers fabricated using a silicon-on-insulator process. A methodology for determining the NIEL and LET coefficients for protons in silicon is presented and total ionizing and non-ionizing absorbed doses calculated. Further work will focus on the irradiation of the fabricated silicon 
cantilevers using proton beams $(E=10,60 \mathrm{MeV})$ with fluences in the range $10^{11}-10^{15} \mathrm{p} / \mathrm{cm}^{2}$ in order to investigate in more detail displacement damages in silicon due to long exposure of silicon-based MEMS devices to protons.

\section{Acknowledgements}

The authors would like to acknowledge European Space Agency, ESA-ESTEC, for the support of this work. Thanks also to Dr Augusto Tazzoli and Prof. Gaudenzio Meneghesso for their help with the irradiations at CN Van de Graff accelerator of the Laboratori Nazionali di Legnaro of the INFN, Italy.

\section{References}

[1] Herbert R. Shea, Radiation sensitivity of microelectromechanical system devices, J. Micro/Nanolith. MEMS MOEMS 8(3), 031303, Jul-Sep 2009.

[2] A.R. Knudson, S. Buchner, P. McDonald, W.J. Stapor, A.B. Campbell, K.S. Grabowski, and D.L. Knies, The effects of radiation on MEMS accelerometers, IEEE transaction on nuclear science, Vol. 43, no. 6, Dec 1996

[3] L. P. Schanwald, J. R. Schwank, J. J. Sniegowski, D. S. Walsh, N. F. Smith, K. A. Peterson, M. R. Shaneyfelt, P. S. Winokur, J. H. Smith, and B. L. Doyle, Radiation Effects on Surface Micromachined Comb Drives and Microengine, IEEE Transaction on nuclear science, Vol. 45, No. 6, Dec 1998

[4] G. J. Papaioannou, V. Theonas, M. Exarchos and G. Konstantinidis, RF sensitivity of MEMS to radiations, 34 Euro pean Microwave Conference - Amsterdam, 2004

[5] L. D. Edmonds, G. M. Swift, C. I. Lee, Radiation response of a MEMS accelerometer: an electrostatic force, IEEE Transaction on Nuclear Science, Vol 45, no. 6, Dec 1998

[6] www.Lemoptix.com

[7] www.SRIM.org

[8] Scott R. Messenger, Edward A. Burke, Robert J. Walters, Jeffrey H. Warner and Geoffrey P. Summers, Using SRIM to calculated the relative damage coefficients for solar cells, Prog. Photovolt. Res. Appl. 2005; 12:115-123

[9] Reynold Panergo, Cheng-Sheng Huang, Chao-Shih Liu, Per G. Reinhall, and Wei-Chih Wang, Resonant Polymeric Waveguide Cantilever Integrated for Optical Scanning, Journal of Lightwave technology, Vol. 25, no. 3, March 2007

[10] Chun-Hyung Cho, Characterization of Young's modulus of silicon versus temperature using a "beam deflection" method with a four-point bending fixture, Current Applied Physics 9, p. 538-545, 2009 